\title{
Global hotspots and correlates of alien species richness across taxonomic groups
}

\author{
Wayne Dawson ${ }^{1 \star}$, Dietmar Moser ${ }^{2}$, Mark van Kleunen ${ }^{3,4}$, Holger Kreft ${ }^{5}$, Jan Pergl ${ }^{6}$, Petr Pyšek ${ }^{6,7,8}$, \\ Patrick Weigelt ${ }^{5}$, Marten Winter ${ }^{9}$, Bernd Lenzner ${ }^{2}$, Tim M. Blackburn ${ }^{10,11,12}$, Ellie E. Dyer ${ }^{10}$, \\ Phillip Cassey ${ }^{13}$, Sally L. Scrivens ${ }^{13}$, Evan P. Economo ${ }^{14}$, Benoit Guénard ${ }^{15}$, César Capinha ${ }^{16,17}$, \\ Hanno Seebens ${ }^{18}$, Pablo García-Díaz ${ }^{13,19}$, Wolfgang Nentwig ${ }^{20}$, Emili García-Berthou ${ }^{21}$, Christine Casal ${ }^{22}$, \\ Nicholas E. Mandrak ${ }^{23}$, Pam Fuller ${ }^{24}$, Carsten Meyer ${ }^{25}$ and Franz Ess| ${ }^{2,8}$
}

\begin{abstract}
Human-mediated transport beyond biogeographic barriers has led to the introduction and establishment of alien species in new regions worldwide. However, we lack a global picture of established alien species richness for multiple taxonomic groups. Here, we assess global patterns and potential drivers of established alien species richness across eight taxonomic groups (amphibians, ants, birds, freshwater fishes, mammals, vascular plants, reptiles and spiders) for 186 islands and 423 mainland regions. Hotspots of established alien species richness are predominantly island and coastal mainland regions. Regions with greater gross domestic product per capita, human population density, and area have higher established alien richness, with strongest effects emerging for islands. Ants and reptiles, birds and mammals, and vascular plants and spiders form pairs of taxonomic groups with the highest spatial congruence in established alien richness, but drivers explaining richness differ between the taxa in each pair. Across all taxonomic groups, our results highlight the need to prioritize prevention of further alien species introductions to island and coastal mainland regions globally.
\end{abstract}

T he transport of species across biogeographic barriers by humans is a key component of global environmental change ${ }^{1-3}$. Some of the species introduced to new regions will establish self-sustaining populations and thus become a persistent part of the local biota ${ }^{4}$. Numbers of these established alien species (EAS) are predicted to increase further as a result of increasing global trade, land-use intensification, urbanization and climate change ${ }^{5}$. Although patterns of EAS richness have been analysed for particular regions ${ }^{6-8}$ and taxa ${ }^{9-13}$ individually, we still lack a global synthesis across a broad range of taxonomic groups. Such a synthesis will be invaluable for (i) identifying geographical hotspots and coldspots of EAS richness, both across and within taxonomic groups, and for (ii) identifying and assessing potential correlates and drivers of EAS richness across different taxonomic groups

Here, we assess global patterns and correlates of EAS richness across eight taxonomic groups by integrating comprehensive published (vascular plants ${ }^{12}$, birds ${ }^{13}$, fishes ${ }^{14}$, ants ${ }^{15}$ and spiders ${ }^{16}$ ) and so far unpublished databases (amphibians, mammals and reptiles) (Fig. 1). As a spatial framework, we use the 609 regions (186 islands or archipelagos, and 423 mainland regions) from level 4 of the Biodiversity Information Standards framework (TDWG) ${ }^{17}$, representing countries or states and provinces within larger countries,

\footnotetext{
'Department of Biosciences, Durham University, South Road, Durham DH1 3LE, UK. ²Division of Conservation Biology, Vegetation and Landscape Ecology, University Vienna, Rennweg 14, A-1030 Vienna, Austria. ${ }^{3}$ Ecology, Department of Biology, University of Konstanz, Universitätsstrasse 10, Konstanz, D-78457, Germany. ${ }^{4}$ Zhejiang Provincial Key Laboratory of Plant Evolutionary Ecology and Conservation, Taizhou University, Taizhou 318000, China. ${ }^{5}$ Biodiversity, Macroecology \& Biogeography, University of Goettingen, Büsgenweg 1, D-37077 Göttingen, Germany. ${ }^{6}$ Institute of Botany, Department of Invasion Ecology, The Czech Academy of Sciences, CZ-25243 Průhonice, Czech Republic. 'Department of Ecology, Faculty of Science, Charles University, Viničná 7, CZ-12844 Prague, Czech Republic. ${ }^{8}$ Centre for Invasion Biology, Department of Botany and Zoology, Stellenbosch University, Private Bag X1, Matieland 7602, South Africa. ' German Centre for Integrative Biodiversity Research (iDiv) Halle-Jena-Leipzig, Deutscher Platz 5e, D-04103 Leipzig, Germany. ${ }^{10}$ Centre for Biodiversity and Environment Research, Department of Genetics, Evolution and Environment, University College London Gower Street, London WC1E 6BT, UK. "Institute of Zoology, Zoological Society of London Regent's Park, London NW1 4RY, UK. ${ }^{12}$ School of Biological Sciences, University of Adelaide, Adelaide, South Australia 5005, Australia. ${ }^{13}$ School of Biological Sciences and Centre for Conservation Science and Technology (CCoST), The University of Adelaide, North Terrace, South Australia 5005, Australia. ${ }^{14}$ Okinawa Institute of Science and Technology Graduate University, 1919-1 Tancha, Onna, Okinawa, 904-0495, Japan. ${ }^{15}$ School of Biological Sciences, The University of Hong Kong, Kadoorie Biological Sciences Building, Pok Fu Lam Road, Hong Kong SAR, China. ${ }^{16} \mathrm{CIBIO} / \mathrm{InBIO}$, Centro de Investigação em Biodiversidade e Recursos Genéticos, Cátedra Infraestruturas de Portugal-Biodiversidade, Universidade do Porto, Campus Agrário de Vairão, P-4485-661 Vairão, Portugal. ${ }^{17}$ Zoologisches Forschungsmuseum Alexander Koenig, Adenauerallee 160, D-53113 Bonn, Germany. ${ }^{18}$ Senckenberg Biodiversity and Climate Research Centre (BiK-F), Senckenberganlage 25, D-60325 Frankfurt am Main, Germany. ${ }^{19}$ Landcare Research, PO Box 69040, Lincoln 7640, New Zealand. ${ }^{20}$ Institute of Ecology and Evolution, University of Bern, Baltzerstrasse 6, CH-3012 Bern, Switzerland. ${ }^{21}$ GRECO, Institute of Aquatic Ecology, University of Girona, 17003 Girona, Catalonia, Spain. ${ }^{22}$ FishBase Information and Research Group, Inc., Khush Hall, International Rice Research Institute, Los Baños, Laguna, Philippines. ${ }^{23}$ Department of Biological Sciences, University of Toronto, 1265 Military Trail, M1C 1A4 Toronto, Ontario, Canada. ${ }^{24}$ United States Geological Survey, Nonindigenous Aquatic Species Program, Wetlands and Aquatic Research Center, 7920 NW 71st Street, 32653 Gainesville, Florida, USA. ${ }^{25}$ Macroecology and Society, German Centre for Integrative Biodiversity Research (iDiv) Halle-Jena-Leipzig, Deutscher Platz 5e, D-04103 Leipzig, Germany. *e-mail: wayne.dawson@durham.ac.uk
} 
and major islands and archipelagos. We identify the global hotspots (high richness) and coldspots (low richness) of EAS across the taxonomic groups while accounting for differences in area and sampling effort. 'Sampling effort' consists of published inventory completeness estimates of native species of amphibians, birds and mammals ${ }^{18}$, vascular plants ${ }^{19}$ and native genera of ants ${ }^{20}$ as a proxy (see Methods). We also explore additional macroecological and socioeconomic correlates behind cross-taxon EAS richness patterns.

We expect regions with higher gross domestic product per capita $(\mathrm{GDPpc})$ or with higher population densities to receive more alien species introductions across taxa (that is, to experience higher colonization pressure through trade and transport), resulting in higher EAS richness ${ }^{7,8,10,21}$. We also test whether EAS richness patterns follow the latitudinal gradients often observed for native biota, with higher richness in regions with higher mean annual temperature and precipitation ${ }^{22,23}$. We expect island regions to have higher EAS richness than mainland regions, as islands are thought to be more prone to the establishment of alien species ${ }^{12,24,25}$. In addition, we expect more isolated oceanic islands to have greater EAS richness, as they have been shown to receive more introductions, at least for birds ${ }^{9}$. We also expect coastal regions (as points of introduction) to have higher EAS richness than landlocked regions. Finally, we assess the degree of spatial congruence of EAS richness among taxonomic groups and explore the variables that might explain differences in spatial species-richness patterns among groups.

\section{Results}

Established alien species richness and its drivers. After accounting for area and sampling effort, hotspot regions for EAS richness across taxonomic groups are predominantly islands, with the top three regions being the Hawaiian Islands, New Zealand's North Island and the Lesser Sunda Islands (Indonesia) (Fig. 2; Supplementary Table 1). The top three coldspots are Antarctica (unsurprisingly), Coral Sea Island Territory (Australasia) and the Laccadive Islands (Supplementary Table 1). Representation of continents differs markedly for regions above and below the richness median $\left(\chi^{2}=16.34\right.$, d.f. $\left.=8, P=0.04\right)$, with the Pacific Islands, Europe and Australasia represented disproportionately among higher-richness regions (Fig. 2; Supplementary Table 2). The model best explaining cross-taxon EAS richness for mainland and island regions includes GDPpc, population density and sampling effort, as well as area, mean annual precipitation, and whether a region is mainland or island(s) (Supplementary Table 3; Table 1). Following the (almost) universal species-area relationship for native species, larger regions have higher EAS richness, but regions with greater GDPpc, higher population density and greater sampling effort for native flora and fauna also have higher EAS richness (Table 1). The effects of climate are less pronounced than those of area, GDPpc and human population density, with a weak trend of higher alien richness in wetter regions (Table 1). Although we only have potential proxy data (GDPpc, population density) for colonization pressure here (that is, the total numbers of species introduced) ${ }^{26}$, our results suggest that cumulative numbers of EAS are driven to a greater extent by differences in area and the pressure of introductions from human history and activity ${ }^{1,3,5,12,21}$ than by climate.

Island regions have, on average, higher cross-taxon EAS richness (mean \pm 1 s.d. proportional cross-taxon richness $=0.17 \pm 0.11$ ) than mainland regions (mean \pm 1 s.d. $=0.11 \pm 0.07$; Table 1 ). In addition, models explaining alien richness of island and mainland regions separately reveal that EAS richness is more strongly related to area, GDPpc and population density on islands than in mainland regions (Table 1). Moreover, EAS richness is strongly related to mean annual temperature for islands, with warmer regions having higher richness, whereas the effect of mean annual temperature on mainland region richness was reversed, with cooler regions having higher richness (Table 1). Mainland regions with better sampled native species also harbour more EAS, but for island regions the effect of sampling effort is weaker (Table 1). Among mainland regions, EAS richness is greater for coastal (mean \pm 1 s.d. proportional cross-taxon richness $=0.13 \pm 0.09$ ) than for landlocked regions (mean \pm 1 s.d. $=0.10 \pm 0.04$ ). Cross-taxon EAS richness on islands tends to be higher for those further from continental landmasses (Table 1).

Taxonomic congruence. The strongest correlations in alien richness between taxonomic groups exist for ants and reptiles (Spearman's $\left.r_{\mathrm{S}}=0.62\right)$, followed by birds and mammals, and vascular plants and spiders (both $r_{\mathrm{S}}=0.55$ ) (Table 2). For ants and reptiles, EAS richness is high in the Hawaiian Islands, southern United States (especially Florida), Madagascar and the Mascarene Islands (Fig. 1b,g). Mammals and birds both have high EAS richness in New Zealand, Hawaiian Islands, Florida and California, and are less congruent in Australia, western Europe and the Caribbean (Fig. 1c,e). Alaska, British Columbia and southern Africa tend to have high EAS richness of mammals, but not birds (Fig. 1c,e). Plants and spiders both have high EAS richness in Hawaii, California and Florida, but are less congruent in other coastal regions of the United States and Canada, and in Australia (Fig. 1f,h). In Europe, the United Kingdom has the highest established alien plant richness, whereas Germany has the highest spider richness (Fig. 1f,h). Overall, the Hawaiian Islands, California, Florida and Australasian regions stand out as having high EAS richness for multiple taxonomic groups (Fig. 1).

The strong correlation in EAS richness between ants and reptiles is reflected in the positive relationship between mean annual temperature and EAS richness of these two taxonomic groups (Fig. 3). For both plants and spiders, human population density shows a strong positive relationship with EAS richness. However, the effects of socioeconomic and macroecological drivers on EAS richness differ markedly between birds and mammals despite the strong correlation between them, with stronger effects of sampling effort for birds (Fig. 3). In addition, EAS richness of fishes, mammals and plants is negatively related to mean annual temperature, despite the relatively weak correlations among them (Fig. 3; Table 2). All other drivers included in best-fitting (lowest corrected Akaike information criterion, AICc) models for each taxonomic group have a positive relationship with EAS richness (Fig. 3).

\section{Discussion}

Our results show that, per unit increase in area, GDPpc and population density, EAS richness increases at a faster rate in island than in mainland regions. A potential reason may be that island regions are more readily invaded by alien species than mainland regions ${ }^{25}$, although a rigorous test of this explanation would require data on failed introductions ${ }^{26}$. The opposing relationships between mean annual temperature and richness for island and mainland regions may result from geographical bias in human activities for islands. Many economically independent island states are tropical, and may have high foreign import volumes (and thus high colonization pressure), whereas most non-tropical islands are part of larger mainland states, with presumably limited foreign trade (and thus low colonization pressure). The opposite may explain the lower EAS richness in tropical mainland regions, which have smaller trade volumes than temperate mainland regions ${ }^{5}$. Higher EAS richness in coastal than landlocked mainland regions is likely to be a consequence of the presence of ports in coastal regions ${ }^{1}$, resulting in overall greater rates of species introductions, as previously shown globally for birds ${ }^{13}$ and for multiple aquatic species in the United Kingdom and Ireland ${ }^{27}$. Despite these effects, substantially more variation (20$26 \%$ more) in EAS richness is explained when also accounting for continent and subcontinent effects (marginal versus conditional $R^{2}$, Table 1). This indicates that EAS richness may be further driven by spatially correlated socioeconomic, historical or political factors not 
a

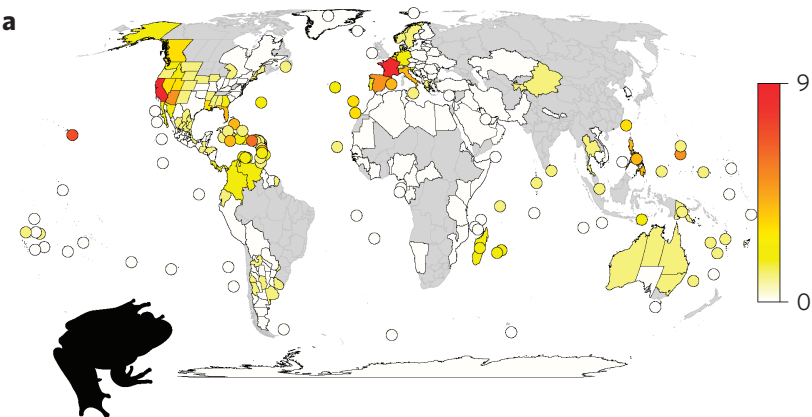

c

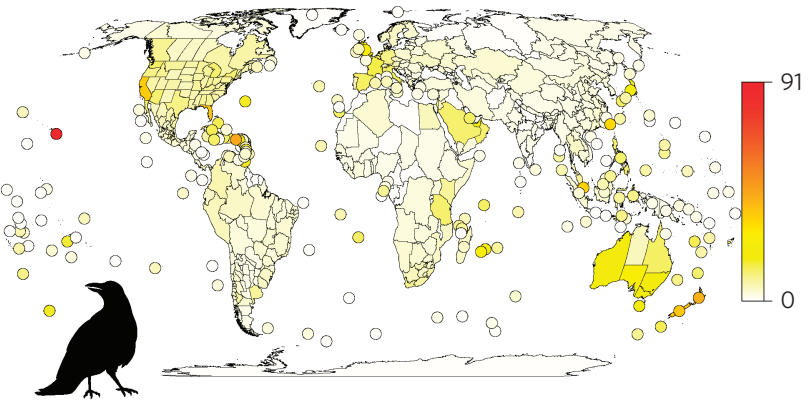

e

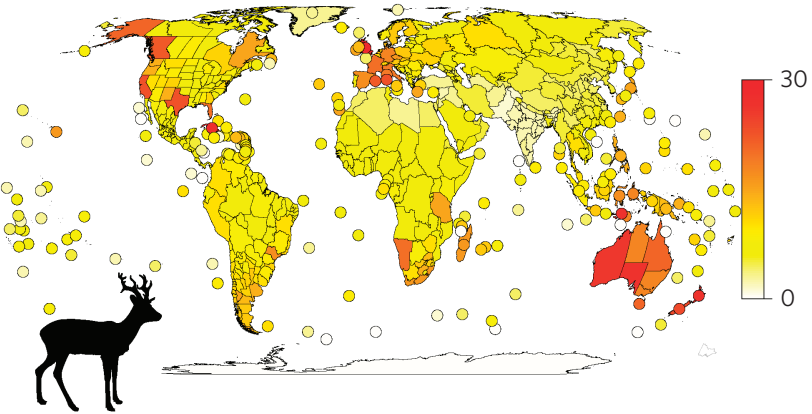

b

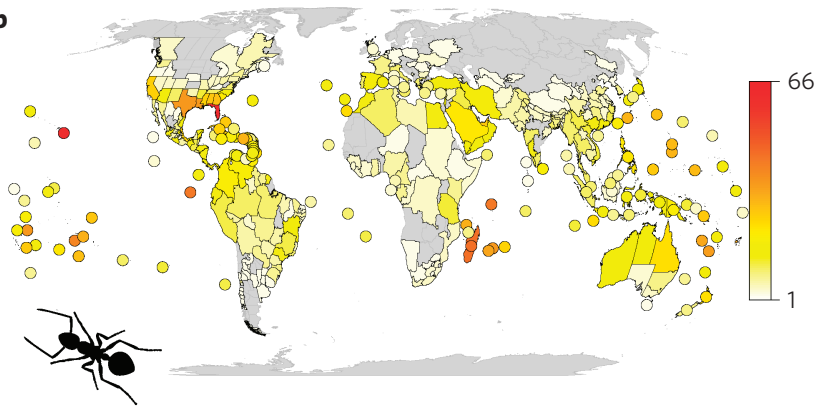

d

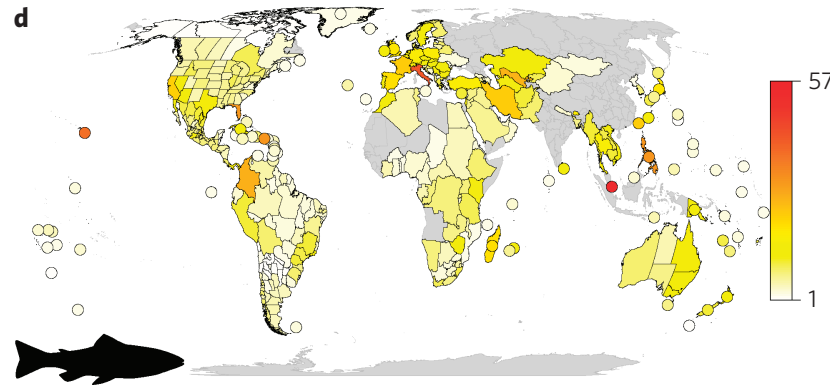

f

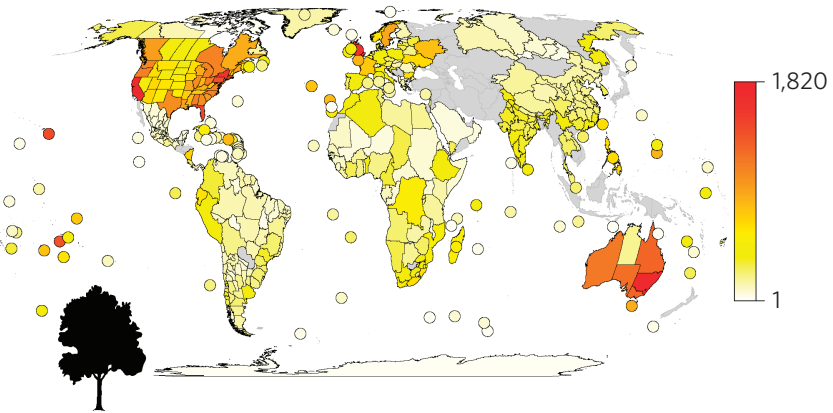

h

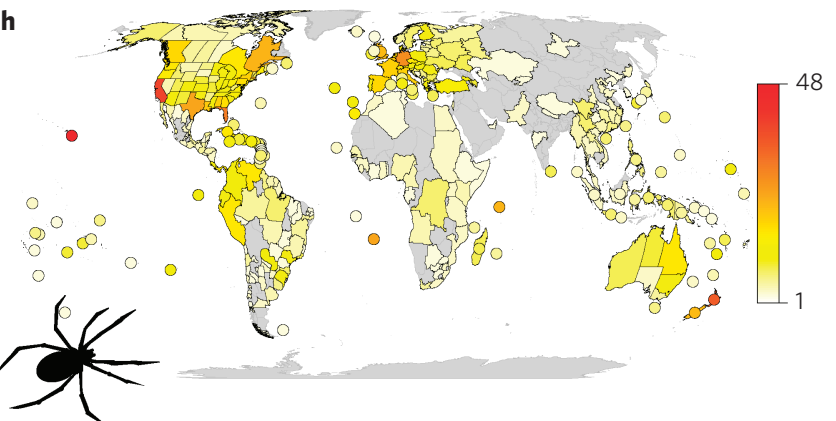

g

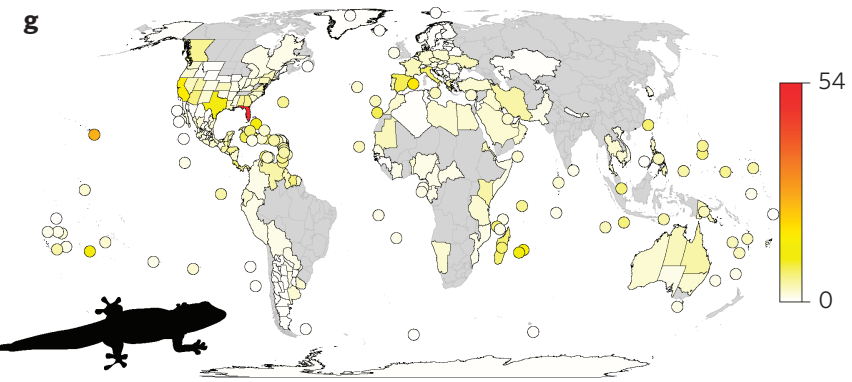

Figure 1 | Established alien species richness in the 609 TDWG level-4 regions with data available per taxonomic group. a, Amphibians. b, Ants. c, Birds. d, Freshwater fishes. e, Mammals. f, Vascular plants. g, Reptiles. h, Spiders. Grey areas represent regions with no data available for a particular taxonomic group. Scales indicate numbers of species.

captured by the variables analysed here. For example, some countries with high GDPpc have recently adopted biosecurity regulations to prevent introductions ${ }^{13}$, and the global spread of the tropical fire ant (Solenopsis geminata) originates from accidental introductions during European colonialism ${ }^{28}$. Such historical and political effects will drive fine-scale variation in colonization pressure ${ }^{26}$, which has been shown to be the strongest determinant of alien bird species richness globally ${ }^{13}$, and for which the socioeconomic information used here may not be a strong proxy.

The moderate-to-strong correlations in EAS richness between birds and mammals, plants and spiders, and ants and reptiles may indicate that EAS richness patterns of these pairs of taxa share similar underlying drivers. Indeed, the models best explaining ant and reptile richness include strong positive effects of mean annual temperature on alien richness (Fig. 3). The correlation between plant and spider richness may reflect horticulture and fruit or vegetable imports acting as important pathways of accidental introductions for both groups ${ }^{29}$. Human population density has similarly strong effects on richness for plants and spiders, but also for fishes, potentially reflecting a greater number of introductions for these groups that have occurred in more densely populated regions (Fig. 3). 
Table 1 | Estimates of models explaining cross-taxon EAS richness for all regions, and for mainland or island regions only.

\begin{tabular}{llll} 
& All regions & Mainland & Island \\
\hline$N$ & 446 & 371 & 75 \\
Intercept & $-1.74(0.08)$ & $-2.32(0.05)$ & $-1.92(0.10)$ \\
Area & $0.35(0.03)$ & $0.25(0.02)$ & $0.53(0.07)$ \\
Sampling effort & $0.17(0.03)$ & $0.17(0.03)$ & $0.10(0.06)$ \\
Area:sampling effort & $0.07(0.02)$ & $0.08(0.01)$ & \\
Mainland & $-0.44(0.07)$ & - & - \\
Coastal & - & $0.13(0.03)$ & - \\
Distance to continent & - & - & $0.18(0.07)$ \\
Mean annual temperature & & $-0.08(0.03)$ & $0.25(0.10)$ \\
Mean annual precipitation & $0.06(0.02)$ & $0.06(0.02)$ & \\
GDP per capita & $0.19(0.03)$ & $0.12(0.03)$ & $0.31(0.07)$ \\
Human population density & $0.23(0.02)$ & $0.19(0.03)$ & $0.35(0.07)$ \\
TDWG continent & 0.14 & 0.07 & 0.22 \\
TDWG level-2 region & 0.24 & 0.20 & 0.31 \\
Residual & 0.30 & 0.26 & 0.40 \\
Marginal/conditional $R^{2}$ & $0.45 / 0.71$ & $0.49 / 0.69$ & $0.58 / 0.79$ \\
\hline
\end{tabular}

Cross-taxon alien richness was natural-log transformed, as were GDP per capita (thousand US\$), human population density $\left(10^{3}\right.$ people $\left.\mathrm{km}^{-2}\right)$ and region area $\left(\mathrm{km}^{2}\right)$. Mean annual precipitatio was square-root transformed. Standard errors are in parentheses. Random effects of TDWG level- 2 regions and continents and residual variation are shown as 1 standard deviation. Cells marked with a dash indicate when a variable was not considered. Marginal $R^{2}$ (variation explained by fixed effects) and conditional $R^{2}$ (fixed and random effects) are also given.

However, spatial congruence of EAS richness may also result from different underlying drivers. For instance, mean annual temperature has a negative effect on established alien plant richness, but is not retained in the model for spider richness (Fig. 3). Similarly, although cooler regions have higher established alien mammal richness, possibly reflecting more frequent transfers of mammals between temperate regions ${ }^{30}$, temperature is unimportant for explaining established alien bird richness (Fig. 3), despite the strong congruence in the richness of these taxa. Although some taxonomic groups show higher spatial congruence than others, no pairwise correlation is above 0.6 , in contrast to native species richness ${ }^{31}$. This indicates that there is a substantial amount of spatial variation in EAS richness among groups (Fig. 1), and as such the effects of key macroecological and socioeconomic correlates will also vary (Fig. 3).

Sampling effort is much more strongly related to established alien bird than to mammal richness, despite their positive correlation (Fig. 3). Historical introductions of birds occurred through the pet trade and Acclimatization Societies ${ }^{13,32,33}$ and may be tightly linked to sampling effort (regions with the best record of native bird richness also tended to have more alien birds) $)^{13}$. Ultimately, completeness of regional inventories, and introduction and recording of alien species could be driven by similar historical, socioeconomic or cultural factors. For instance, regions that have channelled more resources towards creating a more complete record of native species may be the same ones importing and recording more alien species. However, the extent to which current GDPpc and population density reflect colonization pressure and sampling effort in the past is likely to differ among taxa.

Our global assessment of EAS richness and its correlates across eight different taxonomic groups reveals that island regions across the globe, relative to their size and sampling effort, harbour consistently more alien species than mainland regions. Densely populated islands with higher GDPpc and with warmer climates are particularly vulnerable. Coastal mainland regions also have higher EAS richness than landlocked ones. Island and coastal mainland regions may be alien species hotspots because colonization pressure is higher, as has been shown for birds ${ }^{13}$, or because establishment rates among introduced alien species are higher in these regions. Across multiple taxa, it remains to be tested which of these two possibilities is more likely. Socioeconomic factors are clearly important for explaining EAS richness for all taxonomic groups, but effects vary

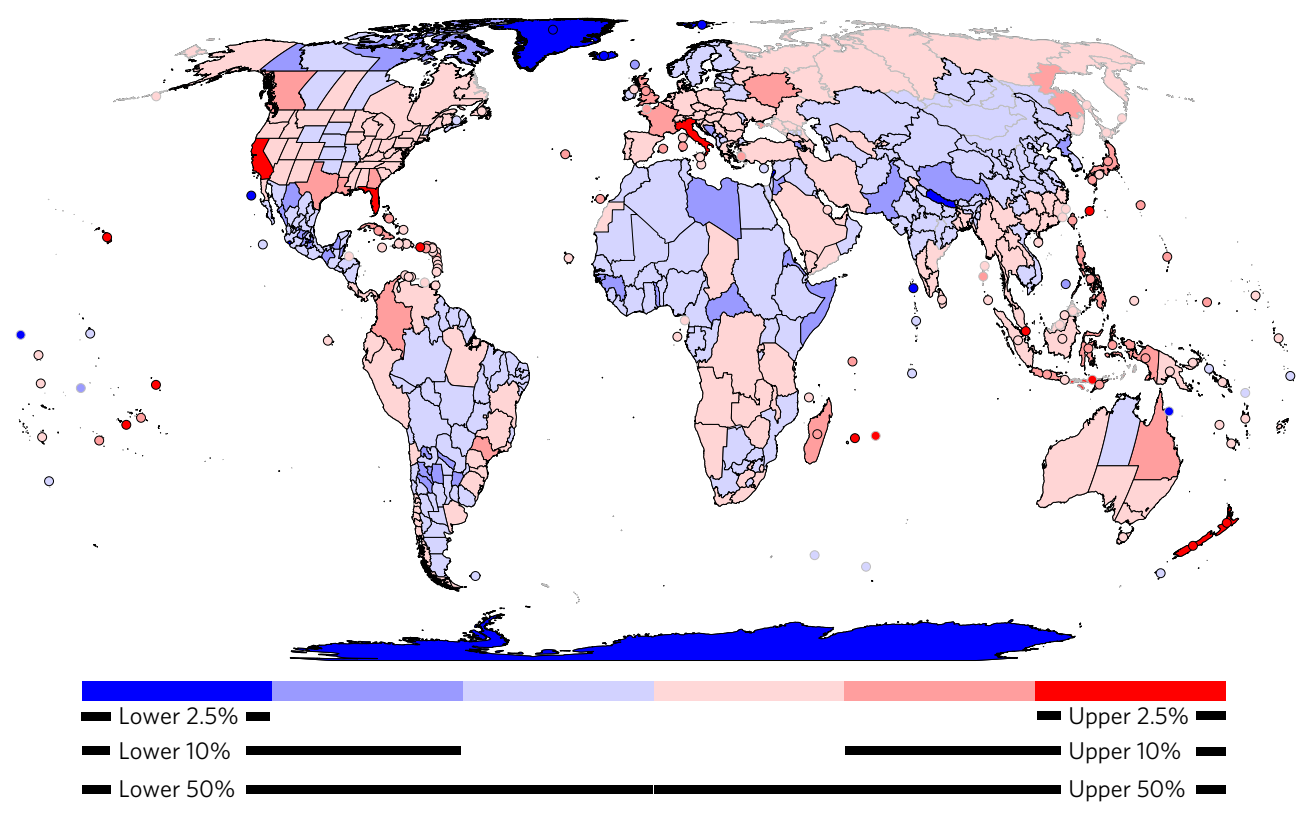

Figure 2 | Hotspot and coldspot regions for cross-taxon established alien species richness across eight taxonomic groups, accounting for area and sampling effort. The taxonomic groups are amphibians, ants, birds, freshwater fishes, mammals, vascular plants, reptiles and spiders. Only TDWG level-4 regions (countries, federal states and islands/archipelagos) with data on sampling effort available were included $(n=534)$. Cross-taxon EAS richness of grey-bordered regions was calculated from three or fewer taxonomic groups, and of black-bordered regions from four or more taxonomic groups. The cross-taxon EAS richness comprises residuals from a linear model, In(cross-taxon alien richness) $\approx$ In(area) $\times$ sampling effort. Upper and lower $2.5 \%$ and $10 \%$ regions are indicated separately from the remaining upper and lower $50 \%$ regions. 
Table 2 | EAS richness correlations (Spearman's $r_{\mathrm{s}}$ ) between pairs of the eight taxonomic groups, for all regions with data available.

\begin{tabular}{|c|c|c|c|c|c|c|c|}
\hline & Ants & Amphibians & Mammals & Birds & Fishes & Plants & Spiders \\
\hline Reptiles & 0.62 & 0.41 & 0.26 & 0.44 & 0.14 & 0.17 & 0.34 \\
\hline Ants & & 0.34 & 0.10 (ns) & 0.29 & 0.15 & 0.06 (ns) & 0.28 \\
\hline Mammals & & & & 0.55 & 0.23 & 0.32 & 0.36 \\
\hline Birds & & & & & 0.34 & 0.39 & 0.46 \\
\hline Fishes & & & & & & 0.35 & 0.40 \\
\hline
\end{tabular}

depending on the group under study. This probably reflects differences among taxonomic groups in how well socioeconomic activity reflects colonization pressure. Ultimately, data on the failed introductions, as well as the successful ones, will enable us further to understand global patterns in EAS richness. Nonetheless, the types of regions most vulnerable to EAS that we have identified should be considered priorities for efforts aimed at preventing further species introductions.

\section{Methods}

Data compilation. Data on EAS richness were obtained from databases for eight taxonomic groups (amphibians, ants, birds, mammals, vascular plants, reptiles and spiders). The data on amphibians, reptiles and spiders and fishes in some regions were compiled specifically for this study. For all taxonomic groups, only EAS outside their native ranges have been considered.
Data on alien vascular plants were obtained from the Global Naturalized Alien Flora database version 1.1, a recently compiled database of 13,168 plant species (including hybrids and subspecies) ${ }^{12}$. The data consist of lists of established alien plant species in 843 geopolitical regions, largely conforming to countries, states, provinces and counties within countries, as well as islands and archipelagos. Data on established alien ants were obtained from the Global Ant Biodiversity Informatics project database, which consists of geographical records of species' presences (accessible through antmaps.org) ${ }^{15}$. Only species records representing establishment in the wild (not in glasshouses or buildings, for example) were included, amounting to 4,061 records for 237 species in total. Data on established alien bird species were obtained from the Global Avian Invasions Atlas $^{34}$, which is a comprehensive global database of 971 alien bird species with 27,723 introduction records at a $1^{\circ}$ grid-cell resolution. Data on 445 established alien mammal species were obtained from a geographically complete mammal database (Alien Mammal Data v1.0), which contains records of established alien mammal species in global administrative areas (GADM; www.gadm.org). Data on 81 established alien amphibian and 203 established alien reptile species were compiled from multiple

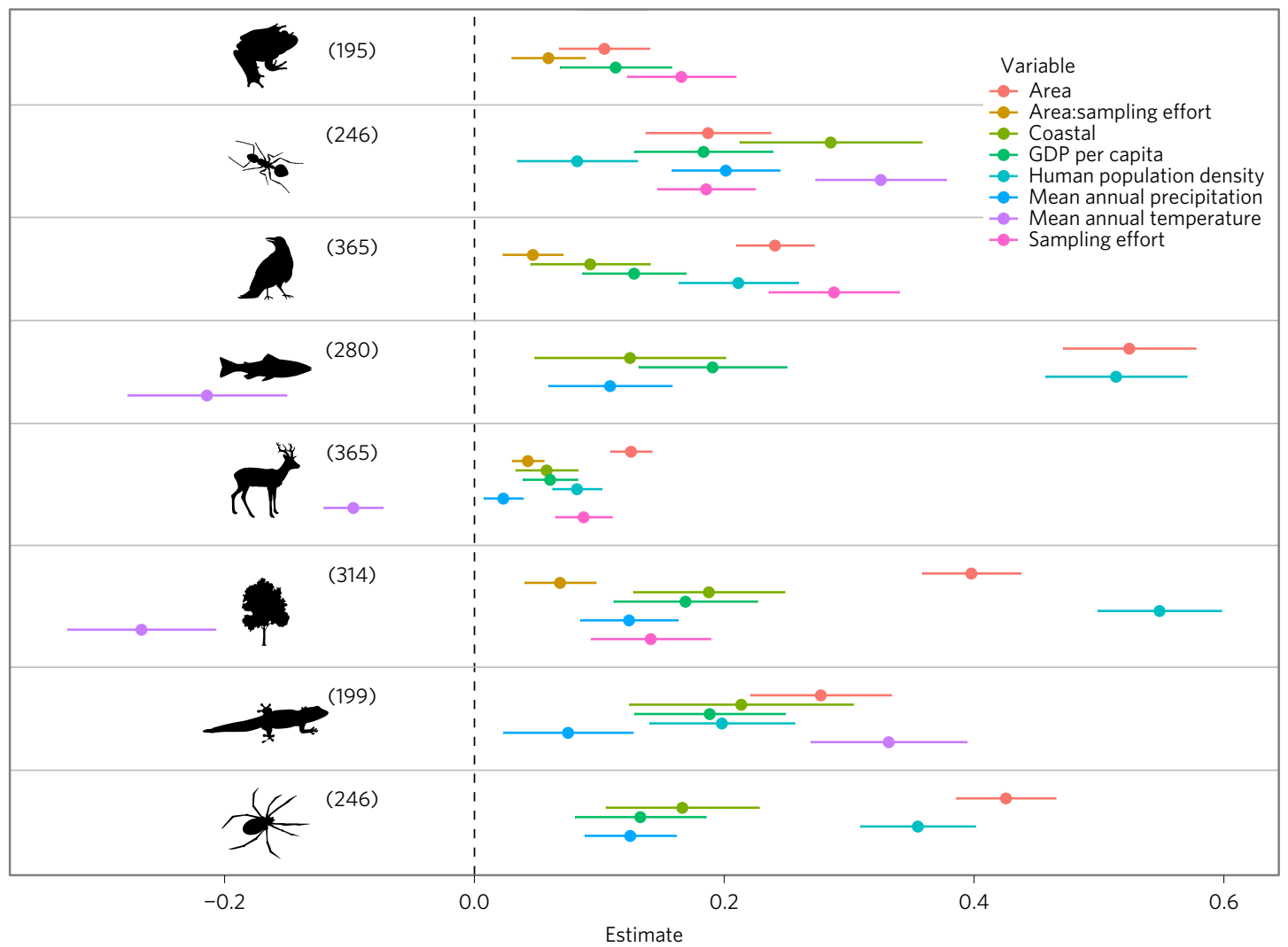

Figure 3 | Effects of area, climate, sampling effort, GDP per capita, human population density and whether a region is coastal on established alien species richness of eight taxonomic groups. The figure is for mainland regions only, owing to insufficient data for islands for all taxonomic groups. Estimates ( \pm 1 standard error) of effects were obtained from linear mixed-effects models of $\ln ($ species richness +1 ), with subcontinental regions (TDWG level 2) nested within continents, as random effects. Note that only variables that were kept in the final models are shown (Supplementary Table 6), and thus numbers of estimates differ between groups. Numbers in parentheses are numbers of regions included per taxonomic group. 
sources into a database, the Global Alien Amphibians and Reptiles Database, by C. Capinha, F.E. and H.S. specifically for this study, and merged with a separate database compiled by P.G.-D. Data on 454 established alien freshwater fish species (including migratory species with marine life-stages, a total of 2,968 records) were taken from the FishBase database ${ }^{14}$ (accessed 13 October 2015), and supplemented for the United States with the US Geological Survey's Nonindigenous Aquatic Species database (compiled by P.F.), Canada (compiled by N.E.M), multiple sources for Mexico ${ }^{35}$, Argentinian, Chilean, Australian, New Zealand ${ }^{36,37}$ and South African provinces (compiled by E.G.-B.) ${ }^{38,39}$, Japan ${ }^{40}$ and Brazil ${ }^{41}$. Finally, 2,138 records on 207 established alien spider species were compiled from a comprehensive literature search by W.N., including the World Spider Catalog ${ }^{16}$ as a major source, and literature available therein.

Because the data for the different taxonomic groups came from different sources and were originally collected at different spatial resolutions, we harmonized all datasets to fit within the Biodiversity Information standards (TDWG) geographic system ${ }^{17}$. This hierarchical system includes as the finest spatial resolution (level 4) a layer of 609 regions, mostly comprising countries, states and provinces of larger countries, and major island groups. Species records for each taxonomic group were assigned to TDWG level-4 regions, and the total number of EAS per taxonomic group within each region was calculated. Global data coverage was highest for birds and mammals (both have all 609 regions), followed by vascular plants (449 regions, $82 \%$ of global ice-free terrestrial area), ants (402 regions, $64 \%$ of area), freshwater fishes (363 regions, $70 \%$ of area), spiders (348 regions, $66 \%$ of area) and, lastly, amphibians and reptiles (311 and 310 regions, $48 \%$ and $47 \%$ of area, respectively).

\section{Estimating sampling effort. Variation in sampling of EAS among regions can} bias the perceived global patterns in alien species richness. However, data directly measuring the degree of sampling effort of EAS recorded in each region are not available. To account for this potential bias, we instead estimated sampling effort for native species of five taxonomic groups: amphibians, ants, birds, mammals and vascular plants. For amphibians, birds and mammals, we obtained global data at a $12,321 \mathrm{~km}^{2}\left(\sim 1^{\circ}\right)$ grid-cell resolution, comprising the percentage completeness of native species inventories for these groups based on occurrence records mobilized through the Global Biodiversity Information Facility (GBIF) ${ }^{18}$. For vascular plants, we obtained data ${ }^{19}$ at the same resolution comprising the percentage completeness of species records in GBIF, as assessed through comparison with independent estimates of native vascular plant richness. For ants, we used region estimates of the percentage of native ant genera missing from records in the Global Ant Biodiversity Informatics project database (these estimates were obtained from models predicting ant diversity and endemicity, and region completeness was calculated as $100 \%$ minus the percentage of missing genera) ${ }^{20}$. We then calculated the average completeness across grid cells for each TDWG level-4 region large enough to overlie most of at least one grid cell (534 regions in total: completeness could not be calculated for 64 island and 11 mainland regions). An average completeness value across the five taxonomic groups was then calculated per region, and used in subsequent analyses of cross-taxon alien richness (see below; Supplementary Fig. 1). When a taxonomic group had native completeness data available, this was used in the single-taxon analyses (see below). Correlations between completeness values for individual taxonomic groups are all significantly positive (Supplementary Table 4), and because vertebrates, invertebrates and vascular plants are included, we are confident that the average completeness across these groups is broadly representative of the missing three taxonomic groups.

Our use of native species inventories as a proxy for sampling effort of EAS recording assumes that there is a linear positive relationship between sampling efforts of alien and of native species. However, the completeness measures are based on data sources that also include records of alien species, and we have no reason to believe that addition of EAS records will differ spatially in a fundamentally different way from native species records. By focusing our sampling effort estimates on native species, we ensure greater statistical independence between the estimates of EAS richness and sampling effort. In addition, completeness of regional inventories and recording of EAS could be driven by ultimately the same factors, such as human history, socioeconomy and culture. So we may expect certain regions not only to have more resources channelled towards creating a more complete recording of native species, but also to be introducing and recording more alien species.

Cross-taxon established alien richness. Species-richness values per region varied greatly among taxonomic groups (Fig. 1), making direct calculation of mean cross-taxon alien richness non-informative. Therefore, alien richness was converted to a relative richness scale, ranging from 0 to 1 , for each taxonomic group, calculated as the regional species richness divided by the maximum richness for a given taxonomic group. The cross-taxon EAS richness for each region was then calculated as the mean of relative richness values across taxonomic groups. The number of taxonomic groups with data available per region ranged from two (46 regions) to the maximum of eight (145 regions), with most regions (503) having four or more taxonomic groups.

As EAS richness of regions may depend on their area and the level of sampling effort in recording alien species, we also calculated a richness value correcting for these effects. This was achieved by fitting a linear model of $\ln$ (cross-taxon
EAS richness) as a function of $\ln \left[\right.$ area $\left.\left(\mathrm{km}^{2}\right)\right]$, interacting with the sampling effort estimate (both variables were centred on their means and scaled to their standard deviations). The interaction was included, as an effect of area may be more detectable for regions with greater sampling effort. Indeed, the interaction term was significant and positive (estimate $[ \pm$ standard error, SE] $=0.102[0.030]$, $\left.F_{1,536}=11.548, P<0.001\right)$, as were the effects of area (estimate $[ \pm \mathrm{SE}]=0.156$ $\left.[0.033], F_{1,536}=15.582, P<0.001\right)$ and sampling effort (estimate $[ \pm \mathrm{SE}]=0.178$ $\left.[0.025], F_{1,536}=53.888, P<0.001\right)$ alone. This model explained $13 \%$ of variation in cross-taxon alien species richness, and a model that explicitly included the number of taxonomic groups as a weighting yielded very similar results. No regions with zero species across taxonomic groups were included owing to a lack of data on sampling effort. The residuals from the non-weighted model were then extracted and used as area- and sampling-effort-corrected, cross-taxon EAS richness for mapping and identifying hotspot regions.

Potential drivers of EAS richness. We explored the ability of several variables to explain variation in cross-taxon EAS richness and richness of individual taxonomic groups. We calculated GDPpc (in US\$) for each TDWG level 4 region as the average of estimated values in $1 \mathrm{~km}^{2}$ grid cells, using estimates derived from night-time light from ref. ${ }^{42}$. Population density in the year 2000 was calculated in a similar manner from $1 \mathrm{~km}^{2}$ grid-cell values obtained from the Global Rural Urban Mapping Project (GRUMP; http://sedac.ciesin.columbia.edu/data/set/grump-v1population-density). Mean annual temperature and mean annual precipitation were downloaded at 1 minute resolution from WORLDCLIM (www.worldclim. org; mean annual temperature $\mathrm{BIO} 1$, and mean annual precipitation $\mathrm{BIO} 12$ from the bioclim variables), and averages were calculated for each TDWG level-4 region. Area $\left(\mathrm{km}^{2}\right)$ was calculated as geodesic area excluding permanent ice-sheets, and each region was classified as being island (including archipelagoes) or mainland (part of a continental landmass; Greenland was coded as an island, Australia was treated as a continental landmass). Distance to mainland was calculated as the geodesic distance to the next continental landmass ${ }^{43}$. Mainland regions were further categorized as being coastal (that is, bordering seas and/or oceans, not including the Caspian Sea) or landlocked.

Analyses. To assess the correlates of cross-taxon EAS richness patterns across all regions, we used linear mixed models using the R package 'nlme'44 (ln-transformed, to satisfy assumptions of normality of residuals and variance homogeneity) as a function of the following fixed effects: area, sampling effort (average percentage native species completeness), and area interacting with sampling effort, gross domestic product per capita (GDPpc), population density, mean annual temperature, mean annual precipitation, and whether a region was a mainland or island region. Area, GDPpc and population density were ln-transformed, and mean annual precipitation was square-root-transformed to linearize relationships with alien richness. We included the TDWG level-2 subcontinental regions, nested within TDWG continents, as random effects (random intercepts only). We also used the same model as above to model ln-transformed cross-taxon EAS richness for mainland and island regions separately, replacing the mainland-island status with, respectively, a binary variable describing mainland regions as landlocked or coastal, and with distance to the nearest continental landmass (km, square-roottransformed) for island regions. We inspected Akaike's Information Criterion corrected for small sample size (AICc) for all full models, and all models nested within them, to identify the set of models within 6 AICc units of the lowest-AIC model that best explain cross-taxon EAS richness for all regions, and for mainland and island regions separately (Supplementary Table 3). The lowest-AICc models explaining richness across all regions, and for mainland and island regions only, were selected for inference, and marginal $R^{2}$ (accounting for fixed effects) and conditional $R^{2}$ (accounting for fixed and random effects) were calculated.

For individual taxonomic groups, we also ran linear mixed models as above to explain EAS richness ( $\ln [$ number of species +1 ] transformation). Owing to insufficient data on sampling effort for several taxa in island regions, we restricted these analyses to mainland regions. Sampling effort for individual taxonomic groups and its interaction with area were included for amphibians, ants, birds, mammals and vascular plants. Sampling effort and its interaction with area were not included in models explaining fish, reptile and spider alien richness because data on native species inventory completeness were not available for these taxonomic groups. Information on model comparisons for individual taxon richness is summarized in Supplementary Table 5.

For all selected models, we checked for spatial autocorrelation of the residuals by constructing correlograms of Moran's $I$ in relation to increasing distance ( 0 to $15,000 \mathrm{~km}$ ) between centroids of neighbouring regions. This was achieved using the 'spline.correlog' function in the R package 'ncf' ${ }^{45}$. To assess significance of spatial autocorrelation, a 95\% confidence interval about Moran's I was constructed from 500 bootstrapped randomizations of the regions' residuals from the models. For models explaining cross-taxon EAS richness, there was a significant but only small amount of autocorrelation (Moran's $I \leq 0.19$ ) when all regions or only mainland regions were considered, and only at very short distances between regions ( $<500 \mathrm{~km}$; Supplementary Fig. 2). For individual taxonomic groups, models showed a significant but small amount of autocorrelation at very short distances for only amphibians (Moran's $I \leq 0.33$, distance $\leq 650 \mathrm{~km}$ ), birds 
(Moran's $I \leq 0.23$, distance $\leq 150 \mathrm{~km}$ ), and freshwater fishes (Moran's $I \leq 0.30$, distance $\leq 750 \mathrm{~km}$ ) (Supplementary Fig. 3).

Congruence in EAS richness among taxonomic groups. The strength of correlation in EAS richness between taxonomic groups was assessed by calculating Spearman correlation coefficients for every combination of pairs of taxonomic groups, using the actual species richness for each taxonomic group. The resulting number of regions used per pairwise correlation is shown in Supplementary Table 6. All analyses were conducted in $\mathrm{R}$ version $3.2 .3^{46}$.

Data availability. The dataset analysed in this study is available in Zenodo data repository, https://zenodo.org/record/556393\#.WPjH08a1s2w.

Received 19 August 2016; accepted 11 May 2017; published 12 June 2017

\section{References}

1. di Castri, F. in Biological Invasions: A Global Perspective (eds Drake, J. A. et al.) 1-30 (Wiley, 1989).

2. Simberloff, D. et al. Impacts of biological invasions: what's what and the way forward. Trends Ecol. Evol. 28, 58-66 (2013).

3. Lewis, S. L. \& Maslin, M. A. Defining the Anthropocene. Nature 519, 171-180 (2015)

4. Blackburn, T. M. et al. A proposed unified framework for biological invasions. Trends Ecol. Evol. 26, 333-339 (2011).

5. Seebens, H. et al. Global trade will accelerate plant invasions in emerging economies under climate change. Glob. Chang. Biol. 21, 4128-4140 (2015).

6. Poessel, S. A. Beard, K. H., Callahan, C. M., Ferreira, R. B. \& Stevenson, E. T. Biotic acceptance in introduced amphibians and reptiles in Europe and North America. Glob. Ecol. Biogeogr. 22, 192-201 (2013).

7. Essl, F. et al. Socioeconomic legacy yields and invasion debt. Proc. Natl Acad. Sci. USA 108, 203-207 (2011).

8. Jeschke, J. M. \& Genovesi, P. Do biodiversity and human impact influence the introduction or establishment of alien mammals? Oikos 120, 57-64 (2011).

9. Blackburn, T. M., Cassey, P. \& Lockwood, J. L. The island biogeography of exotic bird species. Glob. Ecol. Biogeogr. 17, 246-251 (2008).

10. Capinha, C., Essl, F., Seebens, H., Moser, D. \& Pereira, H. M. The dispersal of alien species redefines biogeography in the Anthropocene. Science 348, 1248-1251 (2015)

11. Essl, F., Dullinger, S., Moser, D., Steinbauer, K. \& Mang, T. Macroecology of global bryophyte invasions at different invasion stages. Ecography $\mathbf{3 8}$, 488-498 (2015).

12. van Kleunen, M. et al. Global exchange and accumulation of non-native plants. Nature 525, 100-103 (2015).

13. Dyer, E. E. et al. The global distribution and drivers of alien bird species richness. PLoS Biol. 15, e2000942 (2017).

14. Froese, R. \& Pauly, D. (eds) FishBase v. 09/2015 (2015); http://www.fishbase.org

15. Guénard, B., Weiser, M. D., Gomez, K., Narula, N. \& Economo, E. P. The Global Ant Biodiversity Informatics (GABI) database: synthesizing data on ant species geographic distribution. Myrmecol. News 24, 83-89 (2017).

16. World Spider Catalog Version 17.0 (Natural History Museum Bern, 2015) http://wsc.nmbe.ch

17. Brummit, R. K. World Geographical Scheme for Recording Plant Distributions 2nd edn. (Hunt Institute for Botanical Documentation, 2001).

18. Meyer, C., Kreft, H., Guralnick, R. \& Jetz, W. Global priorities for an effective information basis of biodiversity distributions. Nat. Commun. $\mathbf{6}$, 8221 (2015).

19. Meyer, C., Weigelt, P. \& Kreft, H. Multidimensional biases, gaps and uncertainties in global plant occurrence information. Ecol. Lett. 19, 992-1006 (2016).

20. Guénard, B., Weiser, M. D. \& Dunn, R. R. Global models of ant diversity suggest regions where new discoveries are most likely are under disproportionate deforestation threat. Proc. Natl Acad. Sci. USA 109, 7368-7373 (2012).

21. Pyšek, P. et al. Disentangling the role of environmental and human pressures on biological invasions across Europe. Proc. Natl Acad. Sci. USA 107, 12157-12162 (2010).

22. Gaston, K. J. Global patterns of biodiversity. Nature 405, 220-227 (2000).

23. Lambdon, P. W. et al. Alien flora of Europe: species diversity, temporal trends, geographical patterns and research needs. Preslia 80, 101-149 (2008).

24. Denslow, J. S. Weeds in paradise: thoughts on the invasibility of tropical islands. Ann. Missouri Bot. Gard. 90, 119-127 (2003).

25. Lonsdale, W. M. Global patterns of plant invasions and the concept of invasibility. Ecology 80, 1522-1536 (1999).

26. Lockwood, J. L., Cassey, P. \& Blackburn, T. M. The more you introduce the more you get: the role of colonization pressure and propagule pressure in invasion ecology. Divers. Distrib. 15, 904-910 (2009).

27. Gallardo, B. \& Aldridge, D. C. The "dirty-dozen": socio-economic factors amplify the invasion potential of 12 high-risk aquatic invasive species in Great Britain and Ireland. J. Appl. Ecol. 50, 757-766 (2013).
28. Gotzek, D. et al. Global invasion history of the tropical fire ant: a stowaway on the first global trade routes. Mol. Ecol. 24, 374-388 (2015).

29. Nentwig, W. Introduction, establishment rate, pathways and impact of spiders alien to Europe. Biol. Invas. 17, 2757-2778 (2015).

30. Genovesi, P., Bacher, S., Kobelt, M., Pascal, M. \& Scalera, R. in Handbook of Alien Species in Europe (eds DAISIE) 119-128 (Springer, 2009).

31. Qian, H. \& Ricklefs, R. E. Global concordance in diversity patterns of vascular plants and terrestrial vertebrates. Ecol. Lett. 11, 547-553 (2008).

32. Lever, C. in Encyclopedia of Biological Invasions (eds Simberloff, D. \& Rejmánek, M.) 1-4 (Univ. California Press, 2011).

33. Felemban, H. M. On the exotic birds imported into Jeddah, Saudi Arabia. Zool. Middle East 8, 15-16 (1993).

34. Dyer, E. E., Redding, D. W. \& Blackburn, T. M. The global avian invasions atlas, a database of alien bird distributions worldwide. Sci. Data 4, 170041 (2017).

35. Espinosa-Perez, H. \& Ramirez, M. Exotic and invasive fishes in Mexico. Check List 11, 1627 (2015).

36. McDowall, R. M. The Reed Field Guide to New Zealand Freshwater Fishes (Reed, 2000)

37. NIWA Atlas of NZ Freshwater Fishes (accessed 24 May 2017) https://www. niwa.co.nz/freshwater-and-estuaries/nzffd/NIWA-fish-atlas

38. Ellender, B. R. \& Weyl, O. L. F. A review of current knowledge, risk and ecological impacts associated with non-native freshwater fish introductions in South Africa. Aquat. Invasions 9, 117-132 (2014).

39. Skelton, P. H. A Complete Guide to the Freshwater Fishes of Southern Africa (Southern Book Publishers, 2001).

40. Invasive Species of Japan Database (Environmental Risk Research Center, National Institute for Environmental Studies, Japan, accessed 18 May 2016) https://www.nies.go.jp/biodiversity/invasive/index_en.html

41. Brazil Invasive Alien Species Database (Inter-American Biodiversity Information Network, accessed 17 May 2016) http://i3n.institutohorus.org.br/www

42. Ghosh, T., Powell, R. L., Elvidge, C. D., Baugh, K. E., Sutton, P. C. \& Anderson, S. Shedding light on the global distribution of economic activity. Open Geogr. J. 3, 148-160 (2010).

43. Weigelt, P. \& Kreft, H. Quantifying island isolation - insights from global patterns of insular plant species richness. Ecography 36, 417-429 (2013).

44. Pinheiro, J., Bates, D., DebRoy, S., Sarkar, D. \& R Core Team. nlme: Linear and nonlinear mixed effects models. $R$ Package Version 3.1-128 (2016).

45. Bjornstad, O.N. ncf: Spatial nonparametric covariance functions. $R$ Package Version 1.1-7 (2016).

46. R Core Team R: A Language and Environment for Statistical Computing (R Foundation for Statistical Computing, 2016).

\section{Acknowledgements}

This research benefited from support from the European Commission (COST Action TD1209). The Deutsche Forschungsgemeinschaft supported H.S. (DFG, grant SE 1891/2-1), M.v.K. (KL 1866/9-1) and M.W. (FZT 118), the Austrian Science Foundation supported F.E., B.L. and D.M. (FWF, grant I2086-B16). P.P. and J.P. were supported by the Academy of Sciences of the Czech Republic (no. RVO 67985939), Praemium Academiae award to P.P. and Czech Science Foundation (project no. 14-36079G). C. Capinha was supported by a postdoctoral grant from the Portuguese Foundation for Science and Technology (FCT/MCTES) and POPH/FSE (EC) grant SFRH/BPD/84422/2012. E.G.-B. was supported by the Spanish Ministry of Economy and Competitiveness (projects CGL2013-43822-R and CGL2015-69311-REDT). C.M. was supported by the Volkswagen Foundation through a Freigeist Fellowship.

\section{Author contributions}

The GloNAF core team (M.v.K., P.P., W.D., F.E., J.P., M.W., H.K. and P.W.), T.M.B., H.S. and B.L. conceived the idea; W.D. coordinated data collation, and designed and led the analyses and writing with major inputs from F.E., D.M. M.v.K., P.P., H.K., M.W., J.P. and P.W., and further inputs from all other authors. Data were contributed by the GloNAF database for vascular plants, E.P.E. and B.G. for ants, C. Capinha, F.E., H.S. and P.G.-D. for amphibians and reptiles, T.M.B. and E.E.D. for birds, C. Casal, E.G.-B., P.F. and N.E.M. for fishes, P.C. and S.L.S for mammals, and W.N. for spiders. D.M. collected and calculated data on region area and sampling effort. C.M. contributed data on completeness of native species richness inventories.

\section{Additional information}

Supplementary information is available for this paper.

Reprints and permissions information is available at www.nature.com/reprints. Correspondence and requests for materials should be addressed to W.D.

How to cite this article: Dawson, W. et al. Global hotspots and correlates of alien species richness across taxonomic groups. Nat. Ecol. Evol. 1, 0186 (2017).

Publisher's note: Springer Nature remains neutral with regard to jurisdictional claims in published maps and institutional affiliations.

\section{Competing interests}

The authors declare no competing financial interests. 